\title{
Application of a Novel Modulation Scheme of PS-RZ-QPSK Signal in High-speed Optical Transmission System
}

\author{
Lei Xu ${ }^{1, a}$, Jinnan Zhang ${ }^{1, b}$, Yang'an Zhang ${ }^{1, \mathrm{c}}$, Mi Lin ${ }^{1, \mathrm{~d}}$ \\ State Key Laboratory of Information Photonics and Optical Communications, Beijing University of Posts and \\ Telecommunications, Beijing 100876, China \\ axuleibupt@bupt.edu.cn, ${ }^{\mathrm{b}}$ jinnanjinnan@gmail.com, ${ }^{\mathrm{c}}$ zhang@bupt.edu.cn, ${ }^{\mathrm{d}}$ Imnice1985@gmail.com
}

\begin{abstract}
Modulation principle of dual-polarization quadrature phase shift keying signal and polarization-switched QPSK signal are demonstrated and advantages of PS-QPSK are proved in theoretically. A novel modulation scheme of PS-RZQPSK signal is proposed in this paper. The scheme reduces transmitter cost by less use of Mach-Zehnder modulator, but also presents similar performance as traditional structure for PS-RZ-QPSK. The simulate result indicates PS-RZ-QPSK can achieve a better transmission performance than DP-RZ-QPSK at the same bit rate $(84 \mathrm{~Gb} / \mathrm{s})$ and baud rate $(28 \mathrm{GBd})$, and proves showing the feasibility of novel modulation scheme.
\end{abstract}

Keywords-High-speed Optical Transmission System; polariza tion-switched quadrature phase shift keying; novel Modulation Scheme; coherent detection; dual-polarization quadrature phase shift keying.

\section{INTRODUCTION}

This The interest in high speed coherent optical fiber transmission systems promotes the development of advanced modulation formats. It's important to efficiently compensate the influence of dispersion, polarization mode dispersion and nonlinear effects in high speed transmission system. Digital optical transmission system uses technologies such as error correction encoding, advanced modulation scheme and photoelectric processing technology to restrain the loss $[1,2]$. A suitable format with an effective modulation scheme can effectively reduce the transmission loss and improve the spectral efficiency and transmission capacity. Benefit from improvement of coherent technology, polarization multiplexing technology [3], especially 4-dimensional (4-D) modulation format, is come to the field of view and gets more and more attention in recent years. Among typical 4-D formats, polarization-switched quadrature phase shift keying (PSQPSK) signal shows better sensitivity than dual-polarization QPSK (DP-QPSK) signal.

As discussed in [4-6], PS-QPSK is the most power efficient modulation formats in coherent transmission systems. Compared with binary modulation formats such as binary phase shift keying (BPSK), PS-QPSK has advantages of much narrow spectrum, high spectrum utilization, large dispersion tolerance, low susceptibility to fiber nonlinear effects. In addition, combining PS-QPSK signal with returnto-zero (RZ) modulation, that is PS-RZ-QPSK, will induce the inter-symbol interference (ISI).
This paper proposes a novel modulation scheme for PSRZ-QPSK signal. Traditionally, get PS-RZ-QPSK signal, another Mach-Zehnder (MZ) modulator [7] for RZ modulation need to be cascaded after PS-QPSK transmitter. Our scheme optimizes the approach of PS-RZ-QPSK signal by accomplish phase modulation and RZ modulation in one $\mathrm{MZ}$ modulator, which extremely reduces transmitter cost. According to the simulation analysis, it is confirmed that the performance of novel scheme signal achieves the same magnitude as normal PS-RZ-QPSK signal.

\section{A NOVEL MOdUlAtion SCHEME FOR PS-RZ-QPSK}

In signal space [4], the dimensions of 4-D signal include two orthogonal phases (in phase and quadrature) and two orthogonal polarization states(X mode and $\mathrm{Y}$ mode). A final way to express the signal is as a $4-\mathrm{D}$ vector . DP-QPSK signal has 16 levels, which can be expressed (in normalized units) as . PS-QPSK signal can be expressed as 8 levels , , both of which can be converted from . From these expressions, we can find out the structures for DP-QPSK and PS-QP SK, which will be explained subsequently.

As discussed above, there are two kinds of modulation structure to generate PS-QPSK signal corresponding to C2' and C2". The structure corresponding to C2' implements QPSK modulation with two parallel data bit patterns, and another bit pattern determines whether the optical QPSK signal to be transmitted on $\mathrm{X}$ polarization or $\mathrm{Y}$ polarization. Another structure relies on C2", which can immediately make use of DP-QPSK transmitter after some process on transmitted data.

Fig. 1 shows the structure of DP-QPSK transmitter. Firs tly, a polarization splitter (PBS) is used to split the optical signal into $\mathrm{X}$ polarization and $\mathrm{Y}$ polarization. Then the QPSK modulation [8] is proceed with two parallel MZ modulators for each polarization. A polarization combiner (PBS) combines the two optical signals into one, which is DP-QPSK signal. To get DP-RZ-QPSK signal, another MZ modulator is needed to make RZ modulation on DP-QPSK signal. 


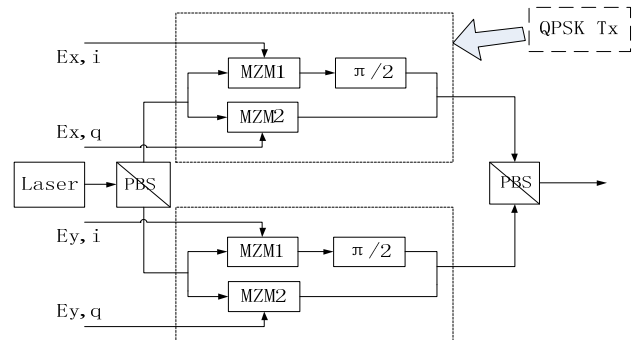

Fig. 1 Block diagram of DP-QPSK transmitter.

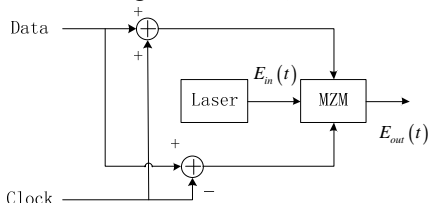

Fig. 2 Block diagram with a single MZ modulator

The According to the analysis above, PS-QPSK signal can be achieved in two ways, as Fig. 3 shows. Fig. 3(a) shows the transmitter corresponding to $\mathrm{C} 2$ '. This paper uses the transmitter in Fig. 3(b), which corresponds to C2".

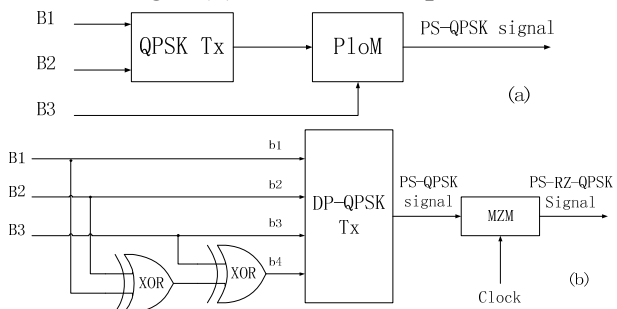

Fig. 3 Block diagram of PS-QPSK modulator. (a) C2'; (b) C2'

Traditionally, RZ modulation is accomplished by cascading a MZ modulator to modulate the amplitude of signal, as showing in Fig. 3(b). This paper proposed a novel scheme of PS-RZ-QPSK signal, whose advantage is less use of $\mathrm{MZ}$ modulator. By setting appropriate parameters and clock signal, one $\mathrm{MZ}$ modulator achieves both phase modulation and amplitude modulation. The structure of novel scheme shows in Fig. 2, and it is called RZ-BPSK Tx in Fig. 4.

The expression of output signal of $\mathrm{MZ}$ modulator [9] is as below.

$$
E_{\text {out }}(t)=E_{\text {in }}(t) \cdot \exp \left(j \frac{\varphi_{1}(t)+\varphi_{2}(t)}{2}\right) \cos \left[\frac{\varphi_{1}(t)-\varphi_{2}(t)}{2}\right]
$$

In which the part of $\exp \left(j \frac{\varphi_{1}(t)+\varphi_{2}(t)}{2}\right)$ changes the phase, and the part of $\cos \left[\frac{\varphi_{1}(t)-\varphi_{2}(t)}{2}\right]$ changes the amplitude.

In the novel scheme, two branches of $\mathrm{MZ}$ modulator are asymmetric, and the input electrical signal of $\mathrm{MZ}$ modulator is mixture of transmitted data signal and clock signal. $V \pi$ is the switching voltage of $\mathrm{MZ}$ modulator, and the clock signal set as , in which Vm is the amplitude of clock and B is the bit rate of each channel. Thus, and in Eq. 1 can be expressed as,

$$
\varphi_{1}(t)=\frac{\pi}{V_{\pi}} \cdot\left[V_{D} \operatorname{Data}(t)+V_{m} \cos (2 \pi B t)+V_{\text {bias }}\right]
$$

$$
\varphi_{2}(t)=\frac{\pi}{V_{\pi}} \cdot\left[V_{D} \operatorname{Data}(t)-V_{m} \cos (2 \pi B t)+V_{\text {bias } 2}\right]
$$

In which Data(t) is the transmitted data ' 0 ' and ' 1 ', and VD is the amplitude of NRZ signal of Data(t). To accomplish phase and amplitude modulation, the parameters should be set as,

$$
\begin{aligned}
& E_{\text {out }}(t)=E_{\text {in }}(t) \cdot \exp \left(j \frac{\pi V_{D}}{V_{\pi}} \cdot \text { Data }(t)\right) \cos \left[\frac{\pi V_{m}}{V_{\pi}} \cos (2 \pi B t)+\frac{\pi}{V_{\pi}} \cdot V_{b}\right] \\
& E_{\text {out }}(t)=E_{\text {in }}(t) \cdot \exp (j \pi \cdot \text { Data }(t)) \cos \left[\frac{\pi}{4} \cos (2 \pi B t)+\frac{\pi}{4}\right]
\end{aligned}
$$

Table 1 shows the relationship of modulation signals and the phase of output optical signals according to Eq. 4 and the QPSK modulator in Fig. 1.

Table 1 Modulation signals and the phase of output optical signals

\begin{tabular}{|l|l|l|l|l|}
\hline $\mathrm{I}$ & 0 & 0 & 1 & 1 \\
\hline $\mathrm{Q}$ & 0 & 1 & 1 & 0 \\
\hline Phase & $\mathrm{j}+1$ & $\mathrm{j}-1$ & $-\mathrm{j}-1$ & $-\mathrm{j}+1$ \\
\hline
\end{tabular}

To demodulate DP-QPSK and PS-QPSK signals, it needs a polarization splitter to split the optical signals into two polarizations, and makes coherent demodulation [10] on each. The scheme of coherent demodulator (Coherent Receiver) shows in Fig. 4.

\section{Simulation AND PERFORMANCE ANALYSIS}

Fig. 4 shows the block diagram of whole system set up in novel scheme. In the simulation system, lasers with linewi$\mathrm{dth}$ of $100 \mathrm{kHz}$ are used as the light source in both transmitter and receiver. The transmitted wavelength is $1552.524 \mathrm{~nm}$. To analyze performance of PS-RZ-QPSK and DP-RZ-QPSK systems at the same bit rate and symbol rate, the experiments on $84 \mathrm{~Gb} / \mathrm{s}$ PS-RZ-QPSK (28GBd for each channel) system, DP-RZ-QPSK (21GBd for each channel) system and $112 \mathrm{~Gb} / \mathrm{s}$ DP-RZ-QPSK (28GBd for each channel) system were implemented.

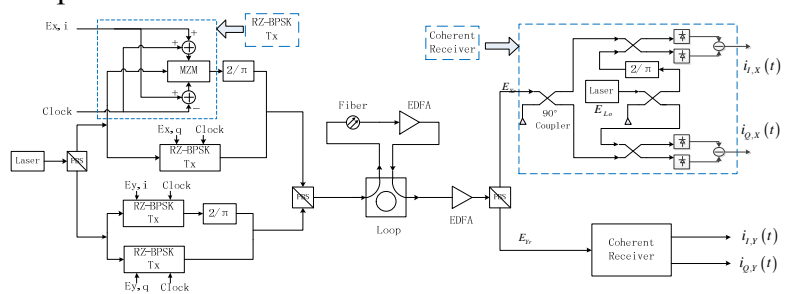

Fig. 4 Block diagram of transmission system of PS-advanced-RZ-QPSK signals.

This paper names the novel scheme system as PSadvanced-RZ-QPSK to distinguish it from traditional PSRZ-QPSK system. For $84 \mathrm{~Gb} / \mathrm{s}$ PS-RZ-QPSK system the three bit patterns Ex,i, Ex,q and Ey,i are pseudo-random binary sequences. The bit pattern Ey,q for the quadrature component of y-polarization is generated as an XORcombination of the other three bit patterns, which described as. For DP-RZ-QPSK system the four bit patterns are pseudo o-random binary sequences at the symbol rate of $21 \mathrm{GBd}$ required for a bit rate of $84 \mathrm{~Gb} / \mathrm{s}$ and $28 \mathrm{GBd}$ for $112 \mathrm{~Gb} / \mathrm{s}$. 
The BER in a back-to-back configuration is shown in Fig. 5. The dashed lines without symbols denote the theoretical limit of BER performance. The symbols are simulated results. For $84 \mathrm{~Gb} / \mathrm{s}$ PS-RZ-QPSK signal, the required OSNR is $11.4 \mathrm{~dB}$ at the BER of $10-3$, which is higher than $0.4 \mathrm{~dB}$ away from the theoretical. The OSNR is $11.7 \mathrm{~dB}$ at BER of $10-3$ for PS-advanced-RZ-QPSK signal, which is just $0.2 \mathrm{~dB}$ higher than the former one. That means the novel scheme makes litter loss on BER performance than normal one. At the same bit rate and same BER, DP-RZ-QPSK signals requires $13 \mathrm{~dB}$ OSNR, which is $0.7 \mathrm{~dB}$ more than theoretical limit. In comparison, PS(-advanced)-RZ-QPSK signals have $1.6 \mathrm{~dB}$ implementation penalty more than DP-RZ-QPSK signals, which is consistent with the theoretical value.

At the same symbol rate (28GBd), PS(-advanced)-RZQPSK signals shows 3dB lower on OSNR $(11.4 \mathrm{~dB})$ than DP-RZ-QPSK signals at the same BER of 10-3. It can be confirmed that PS-RZ-QPSK signals has the best receiver sensitivity, and the novel scheme makes almost same performance as normal ones while less using of $\mathrm{MZ}$ modulators in transmitter.

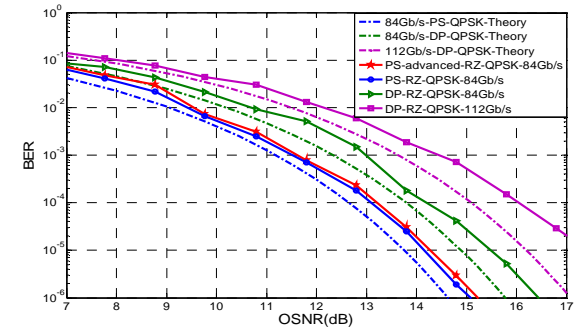

Fig. 5 BER performance of different signals in back-to-back system.

Fig. 6 shows signals' resistance against fiber dispersion and nonlinear effects. In Fig. 6(a), the eye opening penalty (EOP) performance of fiber transmission system at the symbol rate of $28 \mathrm{GBd}$ reflects signals' tolerance to dispersion. It can be informed that PS(-advanced)-RZ-QPSK signal has better EOP performance than DP-RZ-QPSK. PSadvanced-RZ-QPSK signal's EOP is rarely higher than that of normal PS-RZ-QPSK signal.

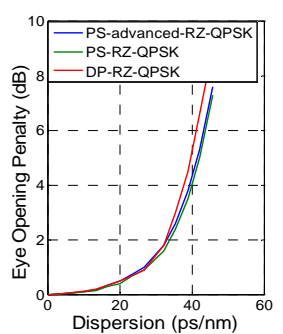

(a) EOP performance;

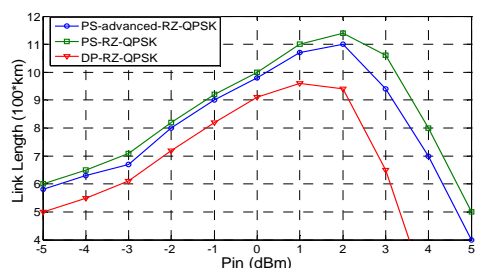

(b) Link length performance $(\mathrm{BER}=10-3)$
Fig. 6 Performance of resistance against dispersion and nonlinear effects.

Component The influence of nonlinear effects on signals can be analyzed by increasing the launch power into the fiber link. In Fig. 6(b), the launch power (Pin) was varied between $-5 \mathrm{dBm}$ and $+5 \mathrm{dBm}$ in step of $1 \mathrm{~dB}$. The link length means the max transmission distance on fiber link at the BER of 10-3. When Pin among $-5 \mathrm{dBm} \sim 1 \mathrm{dBm}$, the link length increases as the launch power increases since nonlinear effects do not obvious. Along with the increasing of launch power, nonlinear effects will show up, making the link length significantly shortened. As showing in Fig. 6(b), the link length is decreased when Pin is at $1 \mathrm{dBm} \sim 5 \mathrm{dBm}$. The optimum launch power resulting in maximum reach is $1 \mathrm{~dB}$ for DP-RZ-QPSK signal and 2dB for PS(-advanced)-RZQPSK signal. That means the PS(-advanced)-RZ-QPSK signal makes better performance on resistance to nonlinear effects than DP-RZ-QPSK signals.

According to the analysis above, it is found that PS(advanced)-RZ-QPSK signal has better receiver sensitivity and provides better performance on resistance to dispersion and nonlinear effects. The proposed novel scheme of PSadvanced-RZ-QPSK signal achieves almost identical performance as normal PS-RZ-QPSK while reducing the cost of transmitter.

\section{CONCLUSION}

This paper theoretical analyzes the implementations of PS-RZ-QPSK, and proposes a novel scheme for PS-RZQPSK signal. The BER performance and influences of dispersion and nonlinear effects on PS(-advanced)-RZ-QPSK and DP-RZ-QPSK signal at the same bit rate and symbol rate are investigated. The results shows that PS(-advanced)-RZQPSK not only benefits from its better sensitivity but also off ers a better tolerance against dispersion and nonlinear effects compared to DP-RZ-QPSK. The novel scheme provides a good quality PS-RZ-QPSK signal while reducing the cost of transmitter by decreasing the amount of MZ modulators.

\section{ACKNOWLEDGMENT}

This work is supported by the fund of young scholar innova-tion project. (2011PTB-00-31), the fund of young scholar innovation project, the fund of young scholar innovation project. (2012RC0406)

\section{REFERENCE}

[1] Xu Xiaogeng, Zhang Xinliang, Liu Deming. Experimental Study on Transmission Performance of Three Different Formats in $40 \mathrm{~Gb} / \mathrm{s}$ Optical Fiber Communication System[J]. CHINESE JOURNAL OF LASERS. 2005,32(10):1371-1376

[2] Wang Xiuge. Research on key technologies of coherent optical communication system[D]. Huazhong University of Science and Technology, 2008,28-72

[3] Wu Ting. Research of polarization multiplexing technology in $80 \mathrm{Gbit} / \mathrm{s}$ optical fiber communication system[D]. Huazhong University of Science and Technology.2009, 21-48

[4] Jinnan Zhang,Yue Gu,Xueguang Yuan,Feng Tian,Xiaoguang Zhang and Jinjing Tao,"A new control algorithm for automatic PMD compensation system", J.Chin. Opt. Lett. , Vol.10, Issue 03 .

[5] M. Karlsson, E. Agrell. Which is the most power-efficient modulation format in optical links?[J]. Opt. Express, 2009,17:10814-10819

[6] Erik Agrell,Magnus Karlsson. Power-Efficient Modulation Formats in Coherent Transmission Systems[J].Journal of Lightwave Technology,2009,27(21/24):5115-5125

[7] Poggiolini P, Bosco G;Carena A. Performance evaluation of coherent WDM PS-QPSK (HEXA) accounting for non-linear fiber propagation effects[J].Optics Express,2010,18(11): 1

[8] Li Jia .Principle of Mach-Zehnder Electro-Optic Modulator and its Applications in Optical Fiber Communication[J]. JOURNAL OF HUNAN INDUSTRY POLYTECHNIC, 2010,10(3):15-17. 
[9] Liu Huiyang, Zhang Xiaoguang, Xu Wei. The principle and simulation of optical DQPSK modulation formats[J].STUDY ON OPTICAL COMMUNICATIONS. 2008,(5):1-3.

[10] Xu Kun, Zhou Guangtao, Wu Jian. Comparisons of High Speed Optical Modulation Formats Using LiNbO3 Optical Waveguide Mod-
ulators[J]. JOURNAL OF BEIJING UNIVERSITY OF POSTS AND TELECOMMUNICATIONS. 2004,27(4):50-54

[11] Betti Silvello, De Marchies Giancarlo, Iannone Eugenio. Coherent Optical Communications Systems[M]. New York : John Wiley \& Sons, 1995, Chapter 3 